\title{
OUTCOMES IN ENDOSCOPIC Sinus SuRgery : OLFACTION, NOSE SCALE AND QUALITY OF Life in A Prospective Cohort Study.
}

Short title

Outcomes and Olfaction in Sinus Surgery

Type of article

Original Article

Authors

Peter Andrews ${ }^{1 凶}$, Anne-Lise Poirrier ${ }^{2}$, Valerie J. Lund ${ }^{1}$ and David Choi ${ }^{3}$.

Affiliation

${ }^{1}$ Department of Rhinology and Facial Plastic Reconstructive Surgery, The Royal National Throat, Nose \& Ear Hospital, 330 Gray's Inn Road, London, WC1X 8DA, United Kingdom. ${ }^{2}$ ENT Department, University Hospital of Liege, Sart-Tilman B35, Liege, Belgium.

${ }^{3}$ Department of Neurosurgery, The National Hospital for Neurology and Neurosurgery, Queen Square, London, WC1N 3BG, United Kingdom.

Keywords

Olfaction, Endoscopic Sinus Surgery, Nasal polyps, Chronic rhinosinusitis 


\section{ABSTRACT}

Objectives: To determine the efficacy of endoscopic sinus surgery on olfactory function in Chronic Rhinosinusitis patients with Nasal Polyps (CRSwNP) and without Nasal Polyps (CRSsNP) and compare nasal obstruction and symptom evaluation (NOSE) scale and quality of life measures before and after surgery.

Design: A prospective cohort study

Setting: Royal National Throat and Nose and Ear Hospital, London UK.

Participants: 113 patients with CRS; 60 CRSwNP and 53 CRSsNP

Outcome measurements: Olfaction was measured using both the University of Pennsylvannia Smell Investigation Test (UPSIT, primary endpoint) and the 'sense of smell' visual analogue score (VAS). The NOSE scale, the SNOT 22 and Lund-Kennedy (LK) scores were also measured pre and post operatively at 6 months.

Results: UPSIT significantly improved after surgery in the CRSwNP subgroup. Baseline UPSIT was significantly better in the CRSsNP subgroup, but the improvement after surgery failed to reach significance in this subgroup. Endoscopic sinus surgery significantly improved the patient's VAS 'sense of smell' in the whole CRS population and in the CRSwNP subgroup. The NOSE, SNOT 22 and LK scores all improved significantly. Improvement measured by UPSIT correlated to SNOT-22, but a correlation between NOSE score and UPSIT was not found.

Conclusions: Endoscopic sinus surgery significantly improved the patient's perceived and measured sense of smell in the CRSwNP subgroup which is the most surgically responsive CRS subgroup. Additionally, improved olfaction in the CRSwNP sub group is likely to improve the patient's quality of life. Endoscopic sinus surgery significantly improved the NOSE scale in both CRS groups at 6 months after surgery. 


\section{INTRODUCTION}

Olfactory dysfunction affects approximately $5 \%$ of the UK population with a prevalence of over $25 \%$ in other published population studies.(1) As a sensory disorder it is more common than blindness and deafness and carries significant psychosocial consequences for the sufferer. (2;3) The two commonest causes for hyposmia/anosmia are viral illnesses and chronic rhinosinusitis (CRS) which account for up to $80 \%$ of all cases, the remaining being due to trauma. (1) The prevalence of CRS in Europe is as high as $10.9 \%$, (4) and the treatment of choice for medical refractory CRS is endoscopic sinus surgery (ESS), with olfactory dysfunction as one of the four major indications, as defined by the European position paper of sinusitis and nasal polyps guidelines (EP3OS).(5) The general role of endoscopic sinus surgery in sinonasal disease is well documented and its function is to reduce nasal obstruction and improve ease of topical steroid application onto the nasal and sinus lining and in doing so potentially improve olfaction. The evidence supporting the efficacy of FESS in the treatment of CRS with or without polyps suggests it is as effective as medical treatment, but not better.(6)

\section{Current evidence supports the positive effects of olfactory improvement following} endoscopic sinus surgery in the treatment of CRS both in the short and long term and up to 5 years post operatively.(7) Additional studies have also shown improved olfactory outcomes following endoscopic sinus surgery albeit not always significant.(8) Systemic and topical corticosteroid in the treatment of hyposmia in chronic rhinosinusitis with nasal polyposis (CRSwNP) have also demonstrated statistically significant improvement in several randomized controlled trials. (9)

CRS subgroup analyses of olfactory outcomes using psychophysical measurements in patients with CRSwNP and CRSsNP have been limited with regards to analysis of quality of 
life outcomes, olfactory function and other patient reported outcome measures (PROMs). A recent study evaluating CRS subgroups following endoscopic sinus surgery demonstrated a significant olfactory improvement in the CRSwNP sub group as measured by Sniffin sticks. This significant improvement was not demonstrated in the CRSsNP subgroup, although as a whole the general CRS population improved.(10) Interestingly this olfactory outcome has not been evaluated using the UPSIT psychophysical olfactory measurement tool.

Up to now, the NOSE scale which is a commonly used PROM in septoplasty and septorhinoplasty surgery, has not been evaluated in the CRS population even though it has been recommended in such patients. $(11 ; 12)$ CRS studies have demonstrated that nasal blockage VAS scores following ESS improved significantly and correlated with improved olfactory function on Sniffin stick evaluation. (13) Quality of life measured by SNOT-22 questionnaire (15) and endoscopic evaluation using the Lund-Kennedy scoring system (16) were previously evaluated in sinus surgery. The question remains whether a specific PROM looking at nasal blockage (NOSE) equally improves following ESS and also correlates with improved olfactory function.

Bugten et al showed that patients with CRSwNP complained more of nasal blockage and a reduced sense of smell whereas patients with CRSsNP complain more of facial pain and headaches, but interestingly they did not find a significant difference in subjective symptom improvement, with both groups responding similarly following FESS.(14)

Our aims were to determine the efficacy of endoscopic sinus surgery (ESS) on olfactory function in CRSwNP and CRSsNP sub groups and to further evaluate the nasal obstruction and symptom evaluation (NOSE) scale in the CRS population.

\section{MATERIALS AND METHODS}


Full NHS Ethical and Research and Development (R\&D) approval were granted for this study. 128 patients were recruited from a dedicated rhinology clinic at the Royal National Throat Nose and Ear Hospital, London, over a 2 year period. The indications for endoscopic sinus surgery (ESS) were in accordance to EPOS guidelines. Exclusion criteria were patients under the age of 16 , pregnancy or patients who were unable to comprehend the assessment.

Olfactory function was assessed subjectively pre- and post-operatively using visual analogue scores and objectively measured using the University of Pennsylvania smell investigation test (UPSIT), a validated psychophysical scratch and sniff test. UPSIT scores were calculated as the number of correct identifications, ranging from 0 to 40 , with 40 representing perfect olfaction. The patient's quality of life was measured using the SNOT-22 questionnaire and their nasal blockage symptoms were measured using the NOSE questionnaire. The patients' nasal cavities were evaluated by the surgeon using the Lund-Kennedy scoring system. All measurements were repeated at 6 months post operatively.

Wilcoxon matched paired tests evaluated the effect of surgery on olfaction (UPSIT, visual analogue scale). Spearman regression was used to assess the correlation between the scores. Results of surgery were dichotomized into two: good result if the SNOT-22 improved by 10 or more and poor outcome in all other cases. Logistic regression was used to examine association between the outcome and UPSIT change after surgery. Effects of co-factors such as smoking, sex and nasal polyposis on baseline UPSIT scores were analysed using the U Mann Whitney test. Linear regression analysis was used to calculate the correlation between age and the baseline UPSIT score. Interaction of co-factors on the olfactory results of sinus surgery was evaluated using 2-ways ANOVA. Statistical tests including receiver operating characteristics were undertaken using Stata version 13.1 (StatCorp, Tx). Graphical 
presentations were performed using GraphPad Prism 5.0a (GraphPad Software, San Diego, California, USA). 


\section{RESULTS \\ Population}

Our population was consistent with the patients undergoing sinus surgery in a tertiary referral hospital (Table 1). Out of the 128 patients for whom inclusion in our study was offered, 113 agreed to enter the study. Half of the patients had chronic rhinosinusitis with nasal polyps. Mean Lund-Mackay score was 10.9 (severe chronic sinusitis on CT scan). Lund-Mackay score was higher in polyps patients (mean $14.9 \pm 5.8$ ) than in patients without polyps (mean $6.4 \pm 5.3$ ). Mean Lund-Mackay score severity was not correlated to the UPSIT baseline score. However, patients with nasal polyps had lower baseline UPSIT score than patients without polyps $(\mathrm{p}<0.0001)$. The UPSIT score was not significantly different in smoking or non-smoking patients in our population. Equally, in our population, age was not correlated to the baseline UPSIT score. The influence of chronic rhinosinusitis on olfaction measured by UPSIT was likely to be stronger than the effect of age and tobacco in our population.

\section{Outcome}

Outcomes are summarized in table 2. The UPSIT score in patients with nasal polyps improved significantly after endoscopic sinus surgery (Wilcoxon $\mathrm{p}=0.0428$ ). Improvement in subjective olfaction scored by visual analogue scale was also seen in our population (Wilcoxon $\mathrm{p}<0.0001$ ) and this improvement was above all observed in the nasal polyps subgroup (Wilcoxon $\mathrm{p}=0.0004$ ). However, in our whole CRS population, olfactory improvement as measured by UPSIT was not significant after surgery. The UPSIT score did not change after surgery in $12.7 \%$ patients improved after surgery in $46.0 \%$ patients and worsened in $41.3 \%$ patients. However, subjective olfaction rated on a visual analogue scale improved in $72.5 \%$ patients, did not change in $13.7 \%$ patients and worsened in $13.7 \%$ 
patients after sinus surgery. The SNOT-22 score, the endoscopic evaluation, the NOSE score and the visual analogue scale improved significantly after surgery in the whole population. The quality of life measured by SNOT-22 improved in $84.5 \%$ patients and decreased in $15.5 \%$ patients after surgery.

When reviewing the subgroups, baseline scores were seen to be worse in the polyp subgroup, but surgical improvement was especially marked in this subgroup. Among polyp patients, $54.5 \%$ improved their UPSIT score, while $12.1 \%$ did not change and $33.3 \%$ worsened after surgery. Subjective olfaction was even better with $81.4 \%$ of polyp patients reporting an improvement on visual analogue scale, $14.8 \%$ patients without change and $3.7 \%$ patients with worsening of olfaction. Improvement of quality of life was observed in $90.9 \%$ polyps patients. The SNOT-22 decreased in $9.1 \%$ polyp patients.

SNOT-22 change and UPSIT change after surgery were correlated. More than $30 \%$ of the SNOT-22 variance was related to UPSIT improvement after surgery in polyps patients $(\mathrm{r} 2=$ 0.379, $\mathrm{p}<0.001$, Figure 1). A correlation between the 'sense of smell' Visual Analogue Score and the UPSIT value was not demonstrated (Spearman R2 $=0.13$ ) A correlation between the NOSE scale and UPSIT score $(\mathrm{r} 2=0.01, \mathrm{p}=0.402)$ equally was not demonstrated. There was no relationship between NOSE change after surgery and UPSIT change after surgery (r2 $=0.223, \mathrm{p}=0.0032$ ).

\section{DISCUSSION}

\section{Synopsis of key findings}

Endoscopic sinus surgery was associated with improved UPSIT 'sense of smell' in the CRSwNP subgroup which represents the most surgically responsive subgroup. Interestingly a 
similar improvement was not demonstrated in the CRSsNP group. Endoscopic sinus surgery improved the patient's perceived 'sense of smell' to a greater extent as measured by the VAS scale in the CRSwNP sub group but not in the CRSsNP group. UPSIT and VAS 'sense of smell' were not exactly correlated. The SNOT 22 outcome equally improved significantly in both subgroups following surgery and importantly a relationship between improved SNOT 22 outcomes and improved UPSIT measurements in the CRSwNP sub group was demonstrated.

This study was the first to evaluate the NOSE scale as an outcome measurement for nasal blockage in the CRS population. The NOSE scale significantly improved in both the CRSsNP and CRSwNP subgroups following endoscopic sinus surgery, although a direct relationship with improved olfaction was not demonstrated. The NOSE questionnaire provides another validated symptom-specific quality of life questionnaire, which specifically assesses the symptom of nasal obstruction and its consequences.

On further sub-group analysis the impact of smoking, age and gender were not shown to affect baseline olfaction nor olfactory function following surgery, suggesting that sinus disease was the most important contributor to impaired olfaction in our cohort, overshadowing other factors. We did not have a single case of anosmia in our surgical cohort and although the majority of our patients were hyposmic prior to surgery and their sense of smell improved post operatively, they still remained hyposmic which is in keeping with current evidence.

\section{Comparison with other studies}

The findings in our study are in agreement with results from a recent study evaluating olfactory outcomes in the CRSwNP subgroup. A significant olfactory improvement in CRSwNP but not in CRSsNP was demonstrated using the Sniffin Stick identification test.(10)Shriever et al in 2013 looked at the effects of nasal surgery, including both sinus 
surgery and septal surgery, on olfaction using the 16 item odor identification 'Sniffin' stick test. At 3.5 months post operatively there was a significant improvement in the sinus surgery arm as opposed to a non-significant improvement in the septal surgery arm and interestingly they both became non-significant at 12 months. They also found that polyps and eosinophilia were good prognostic factors for olfactory outcome improvement. Conversely, in the nonpolyp group, the improvement was not significant (15). Rowe-Jones et al performed a 5 year prospective study of post FESS CRS patients of whom $75 \%$ were CRSwNP and demonstrated a significant improvement in measured olfaction at 2 years post surgery which became non significant at 5 years. Rudmik and Smith performed a literature review on the efficacy of FESS in CRS-related olfactory dysfunction and concluded the evidence supporting its efficacy is equivocal and not significant. (16). Lund et al demonstrated a significant improvement in olfactory function following ESS.(17)

Pade et al demonstrated an improvement in the sense of smell, as measured by Sniffin sticks in $23 \%$ of their post operative patients, no change was seen in $68 \%$, and a decreased function was seen in $9 \%$ of the patients. In terms of the sense of smell, nasal surgery resulted in the highest success rates in patients with eosinophilia and a high degree of polyposis.(18) Dealank et al demonstrated post-endoscopic sinus surgery olfactory improvements in $70 \%$ of their CRS population and olfaction changed for the worse in $8 \% .(19)$

In our study, measured UPSIT olfactory function did not change after surgery in $12.7 \%$ patients, improved after surgery in $46.0 \%$ patients and worsened in $41.3 \%$ patients. Interestingly on subjective olfaction measurements, improvement was seen in $72.5 \%$ patients, did not change in $13.7 \%$ patients and worsened in $13.7 \%$ patients after sinus surgery. This mismatch between the UPSIT and VAS olfactory scores relates to patients with improved 
subjective olfactory preception but worse objective olfactory function. With the use of Sniffin sticks, Pade and Hummel deemed an olfactory improvement of three or more points as a significant change and this may account for the difference in the UPSIT measurements.

Minovi et al, when analyzing olfactory outcome predictors for nasal surgery, found the highest success rates were in women, patients with a high degree of polyposis, and aspirinintolerance. Neither age, presence of asthma, nor the number of preoperative surgical interventions had a significant impact on the outcome of surgery in terms of chemosensory function. (20) However there is increasing evidence supporting early surgical intervention, in medically refractory CRS, with higher post operative healthcare needs exhibited when the delay for surgery is longer.(21)

\section{Clinical applicability of the study}

Our study has highlighted that the CRSwNP subgroup represents the most surgically responsive subgroup of CRS patients for olfactory improvement and will enable better informed consent for patients. Also according to our results, better quality of life is more likely to occur with improved olfactory function in the CRSwNP subgroup. This would imply that olfactory impairment in CRS is probably more complex than just a conductive problem and would also explain why there is a lack of a relationship with the NOSE scale outcome. According to our data, up to one third of the SNOT-22 variance was related to UPSIT change, whereas theoretically, as olfaction is only one of the 22 questions it should be $1 / 22$. This implies that impaired olfaction has a greater impact on quality of life than expected.Conversely, there is a significant proportion of patients whose measured and 
perceived sense of smell becomes worse after surgery, which needs to be explained at the time of informed consent. However, there were no cases of post operative anosmia.

\section{Study Limitations}

One of the limitations of this study is the lack of pre-operative stratification of severity and duration of hyposmia, thus preventing a sub-group analysis of olfactory improvement for these factors. Also UPSIT evaluation is potentially not sensitive enough for complete olfactory detection as it only measures identification as opposed to threshold and discrimination and remains a subjective measure. The objective olfactory outcome measurements include event-related olfactory potentials which require precise odorant delivery via an olfactometer. Equally we did not correlate the NOSE scale outcome to an objective measure of nasal airflow such as Nasal Inspiratory Peak Flow (NIPF) which could be incorporated in future studies. 


\section{CONCLUSION}

Patients' perceived and measured sense of smell in the CRSwNP subgroup improves significantly following ESS and represents the most surgically responsive CRS subgroup. Additionally, we have demonstrated that improved olfaction in the post surgery CRSwNP sub group is more likely to also improve the patient's quality of life. The NOSE scale is a sensitive outcome measure in the CRS population although it does not interact with olfaction.

\section{CONFLICT OF INTEREST}

No conflict of interest to disclose. 


\section{Reference List}

(1) Croy I, Nordin S, Hummel T. Olfactory disorders and quality of life--an updated review. Chem Senses 39[3], 185-194. 2014.

(2) Philpott CM, Boak D. The impact of olfactory disorders in the United kingdom. Chem Senses 39[8], 711-718. 2014.

(3) Lange B, Holst R, Thilsing T, Baelum J, Kjeldsen A. Quality of life and associated factors in persons with chronic rhinosinusitis in the general population: a prospective questionnaire and clinical cross-sectional study. Clin Otolaryngol 38[6], 474-480. 2013

(4) Hastan D, Fokkens WJ, Bachert C, Newson RB, Bislimovska J, Bockelbrink A, et al. Chronic rhinosinusitis in Europe-an underestimated disease. A GA(2)LEN study. Allergy 66(9):1216-1223.[9], 1216-1223. 2011.

(5) Fokens WJ, Lund VJ, Mullol J, Bachert C, et al. EPOS 2012: European position paper on rhinosinusitis and nasal polyps 2012. A summary for otorhinolaryngologists. rhinology 50(1), 1-12. 2012.

(6) Ragab SM, Lund VJ, Scadding G. Evaluation of the medical and surgical treatment of chronic rhinosinusitis: a prospective, randomised, controlled trial. Laryngoscope. 2004 May. Laryngoscope. 114[5], 923-930. 2004.

(7) Rudolf Briner H, Jones N, Simmen D. Olfaction after endoscopic sinus surgery: longterm results*. Rhinology 50, 178-184. 2012.

(8) DeConde AS, Mace JC, Alt JA, Schlosser RJ, Smith TL, Soler ZM. Comparative effectiveness of medical and surgical therapy on olfaction in chronic rhinosinusitis: a prospective, multi-institutional study. Int Forum Allergy Rhinol 4[9], 725-733. 2014.

(9) Golding-Wood DG, Holmstrom M, Darby Y, Scadding GK, Lund VJ. The treatment of hyposmia with intranasal steroids. . Journal of Laryngo.\& Oto 110, 132-135. 1996.

(10) Lind H, Joergensen G, Lange B, Swendstrup F, Kjeldsen AD. Efficacy of ESS in chronic rhinosinusitis with and without nasal polyposis: a Danish cohort study. Eur Arch Otorhinolaryngol June ahead of print. 2015. 
(11) Stewart MG, Witsell DL, Smith TL, Weaver DL, Yueh B, Hannley MT. Development and validation of the Nasal Obstruction Symptom Evaluation (NOSE) scale. Otolaryngol Head Neck Surg 130[2], 157-163. 2004.

(12) Andrews PJ, Choudhury N, Takhar A, Poirrier AL, Jacques T, Randhawa PS. The need for an objective measure in septorhinoplasty surgery: Are we any closer to finding an answer? Clin Otolaryngol 2015; 40:698-703.

(13) Hox V, Callebaut I, Bobic S, Jorissen M, Hellings PW. Nasal obstruction and smell impairment in nasalpolyp disease: correlation between objective and subjective parameters*. Rhinology. 48[426], 432. 2010.

(14) Bugten V, Nordgard S, Romundstad P, Steinsvag S. Chronic rhinosinusitis and nasal polyposis; indicia of heterogeneity. Rhinology 46[1], 40-44. 2008.

(15) Schriever VA, Gupta N, Pade J, Szewczynska M, Hummel T. Olfactory function following nasal surgery: a 1-year follow-up. Eur Arch Otorhinolaryngol 270[1], 107-111. 2013.

(16) Rudmik L, Smith TL. Olfactory improvement after endoscopic sinus surgery. Curr Opin Otolaryngol Head Neck Surg 20[1], 29-32. 2012.

(17) Lund VJ, Scadding GK. Objective assessment of endoscopic sinus surgery in the management of chronic rhinosinusitis: an update. J Laryngol Otol 108[9], 749753. 1994.

(18) Pade J HT. Olfactory function following nasal surgery. Laryngoscope 2008;118(7):1260-4.

(19) Delank.K.W., Stoll W. Olfactory function after functional endoscopic sinus surgery for chronic sinusitis. Rhinology 36[1], 15-19. 1998.

(20) Minovi A, Hummel T, Ural A, Draf W, Bockmuhl U. Predictors of the outcome of nasal surgery in terms of olfactory function. Eur Arch Otorhinolaryngol 265[1], 57-61. 2008.

(21) Hopkins C, Andrews P, Holy CE. Does time to endoscopic sinus surgery impact outcomes in chronic rhinosinusitis? Retrospective analysis using the UK clinical practice research data. Rhinology. 53[1], 18-24. 2015. 
Figure 1

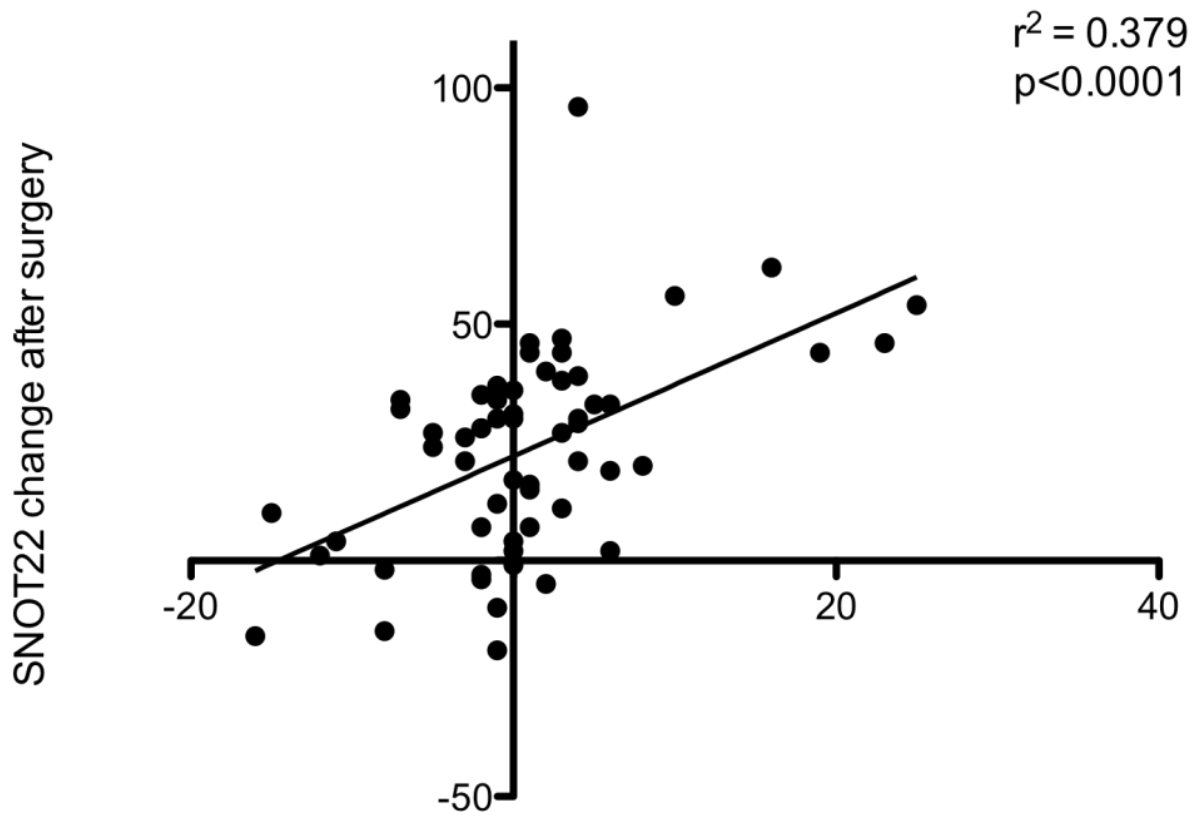

UPSIT change after surgery

Table 1

\section{Patients Data}

\begin{tabular}{l|l|l}
\hline \multirow{2}{*}{$\begin{array}{l}\text { Demographic } \\
\text { Data }\end{array}$} & Total, No & 113 \\
\cline { 2 - 3 } & Age, mean $( \pm \mathrm{sd}), \mathrm{yr}$ & $46.3( \pm 14.9)$ \\
\cline { 2 - 3 } & Sex, No $(\%)$ & $48 \%(42.5 \%)$ \\
& & $65(57.5 \%)$ \\
\hline Clinical Data & Polyposis, No (\%) & $60(53.1 \%)$ \\
& Revision surgery, No (\%) & $13(11.5 \%)$ \\
& Smoking, No (\%) & $24(21.2 \%)$ \\
\hline CT-scan & Lund-Mackay score, mean $( \pm \mathrm{sd})$ & $10.9( \pm 6.9)$ \\
\hline
\end{tabular}


Table 2

Outcome measures

pre-operative post-operative

$\mathrm{p}$

\begin{tabular}{|c|c|c|c|c|}
\hline \multirow{3}{*}{$\begin{array}{l}\text { UPSIT } \\
\text { (value) }\end{array}$} & Whole population, mean $( \pm$ sd) & $25.5( \pm 9.9)$ & $26.6( \pm 9.7)$ & $0.38 \mathrm{~ns}$ \\
\hline & CRSsNP & $29.5( \pm 7.8)$ & $29.1( \pm 8.1)$ & $0.34 \mathrm{~ns}$ \\
\hline & CRSwNP & $21.9( \pm 10.4)$ & $24.3( \pm 10.5)$ & $0.04 *$ \\
\hline \multirow[t]{3}{*}{ NOSE score } & Whole population, mean $( \pm$ sd) & $61.2( \pm 30.6)$ & $27.8( \pm 25.4)$ & $<0.01 * * *$ \\
\hline & CRSsNP & $54.2( \pm 32.7)$ & $31.8( \pm 27.2)$ & $0.01 *$ \\
\hline & CRSwNP & $67.2( \pm 27.7)$ & $22.7( \pm 4.7)$ & $<0.01 * * *$ \\
\hline \multirow{3}{*}{$\begin{array}{l}\text { SNOT-22 } \\
\text { score }\end{array}$} & Whole population, mean $( \pm$ sd) & $53.4( \pm 24.8)$ & $28.9( \pm 23.8)$ & $<0.01 * * *$ \\
\hline & CRSsNP & $52.8( \pm 26.3)$ & $32.5( \pm 25.4)$ & $<0.01 * * *$ \\
\hline & CRSwNP & $54.0( \pm 23.7)$ & $25.8( \pm 22.2)$ & $<0.01 * * *$ \\
\hline \multirow{3}{*}{$\begin{array}{l}\text { Lund- } \\
\text { Kennedy } \\
\text { score }\end{array}$} & Whole population, mean $( \pm$ sd) & $4.6( \pm 2.7)$ & $2.1( \pm 1.7)$ & $<0.01 * * *$ \\
\hline & CRSsNP & $3.2( \pm 2.3)$ & $2.1( \pm 1.4)$ & $0.15 \mathrm{~ns}$ \\
\hline & CRSwNP & $5.7( \pm 2.6)$ & $2.1( \pm 1.8)$ & $<0.01 * * *$ \\
\hline \multirow{3}{*}{$\begin{array}{l}\text { VAS score } \\
\text { on overall } \\
\text { nose sympt }\end{array}$} & Whole population, mean ( \pm sd) & $6.6( \pm 2.8)$ & $3.4( \pm 2.8)$ & $<0.01 * * *$ \\
\hline & CRSsNP & $6.3( \pm 2.7)$ & $4.4( \pm 2.8)$ & $0.04 *$ \\
\hline & CRSwNP & $6.9( \pm 2.8)$ & $2.4( \pm 2.5)$ & $<0.01 * * *$ \\
\hline \multirow{3}{*}{$\begin{array}{l}\text { VAS score } \\
\text { on sense of } \\
\text { smell }\end{array}$} & Whole population, mean $( \pm$ sd) & $6.0( \pm 3.5)$ & $3.7( \pm 3.4)$ & $<0.01 * * *$ \\
\hline & CRSsNP & $4.4( \pm 3.5)$ & $2.5( \pm 3.0)$ & $0.05 \mathrm{~ns}$ \\
\hline & CRSwNP & $7.4( \pm 2.8)$ & $5.0( \pm 3.4)$ & $<0.01 * * *$ \\
\hline
\end{tabular}

\section{Legends}

Figure 1

Comparison of SNOT-22 change (Y-axis) and UPSIT change after surgery (Y-axis) in

CRSwNP. SNOT-22 change and UPSIT change after surgery were fairly correlated, and more than $30 \%$ of the SNOT-22 variance was related to UPSIT improvement after surgery. 